\title{
Evaluation of Acute Abdominal Pain Diagnosis using CT \& MRI
}

\author{
Elhadi Araibi, Rajab M. Ben Yousef, Maryem M. Hemair, Fuzi M. Elkut
}

\begin{abstract}
Acute abdominal pain can be evoked by a wide range of abdominal abnormalities, including acute appendicitis, diverticulitis, cholecystitis, and bowel obstruction. Imaging plays an important role in the treatment management of patients because clinical evaluation results can be inaccurate. Different radiological techniques vary in their sensitivity and accuracy for detecting each pain-caused abdominal disorder. To evaluate the diagnostic value of these radiological techniques in detecting the cause of abdominal pain, comparison between MRI and CT scan was made. The study was conducted at Tripoli Medical Center in 2016 on a group of fifteen patients, who diagnosed with an acute abdomen and had an erect and supine abdominal $X$-ray, were studied; including six females and nine males with their age range from thirty-one up to sixty-six years old. All of them recommended to have an abdominal CT examination, and then they recommended also by their doctors to have an abdominal MRI for the final diagnosis, because the abdominal $X$-ray result does not give them enough information about those cases as the result sometimes was looks like normal.

In this study, we found: In eight cases $(53.33 \%)$ of acute abdomen, magnetic resonance imaging (MRI) gave more information than computed tomography scans (CT), In three cases $(20 \%)$ of acute abdomen, computed tomography scans (CT) gave more details than magnetic resonance imaging (MRI). In four cases (26.66) of acute abdomen, the magnetic resonance imaging (MRI) and computed tomography scans (CT), are gave the same information or details. In one case $(6.66 \%)$, the abdominal $\mathrm{X}$-ray result, and the computed tomography scan (CT) results were similar.

Conclusion: MRI can find changes in the structure of organs or other tissues. It also can find tissue damage or disease, such as infection or a tumor. MRI scan can sometimes find a problem in a tissue or an organ that is not seen by X-ray, ultrasound, or CT scan, even when the size and shape of the tissue or organ looks normal. And sometimes MRI test results may be different than those from CT, ultrasound, or X-ray tests because the MRI scan is more specific.
\end{abstract}

Index Terms - Acute abdominal pain, Computerized Tomography, Magnetic Resonance Imaging, abdominal tumors.

\section{INTRODUCTION}

Acute abdominal pain is a common presentation in the outpatient setting, constituting $4 \%-5 \%$ of all patients who present to emergency department (1). Acute abdominal pain represents conditions ranging from benign to life threatening. Acute appendicitis, diverticulitis, cholecystitis, and bowel obstruction are common causes of acute abdominal pain. Other important but less frequent conditions that may cause

Elhadi Araibi, Faculty of Medical Technology, University of zawia Rajab M. Ben Yousef, Faculty of Medical Technology, University of zawia Maryem M. Hemair, Faculty of Medical Technology, University of zawia Fuzi M. Elkut, Faculty of Health Sciences, Elmergib Univesity acute abdominal pain include perforated viscus and bowel ischemia and tumors. Acute abdominal pain is defined as severe pain of more than 6 hours' duration in a previously healthy person that requires timely diagnosis and aggressive treatment, frequently surgical. A subgroup of patients with acute abdominal pain will have a so-called acute abdomen (also known as an acute surgical abdomen). This is restricted to those with an acute presentation of abdominal pain coupled with serious morbidity, a tender abdomen and marked rigidity on abdominal physical examination.

Although the patient history, physical examination, and laboratory test results can narrow the differential diagnosis, imaging is often required for definitive diagnosis and treatment. The American College of Radiology has developed clinical guidelines, the Appropriateness Criteria, based on the location of abdominal pain to help physicians choose the most appropriate imaging study. Currently, computed tomography (CT) and Magnetic Resonance Imaging (MRI) are the cross-sectional imaging modalities of choice in this setting (2).Imaging plays an essential role in narrowing the differential diagnosis and directing management.

Conventional radiography is commonly the initial imaging examination performed in the diagnostic work-up of patients who present with acute abdominal pain to the emergency department. The accuracy values for conventional radiography in the diagnostic work-up of patients with acute abdominal pain are not convincing.Thus, the use of conventional radiography might justifiably be limited to these patients only, especially if CT is not available. In the majority of patients, further imaging is warranted after conventional radiography. US and CT (3), as compared with conventional radiography, yield markedly higher accuracy values. Ultrasound, CT and MRI all have various advantages and disadvantages over each other and each has its roles, which may not often interchangeable.

CT scanner became a clinical reality in the early 1970's. In 1979, Cormack shared the Nobel Prize in Medicine and Physiology with Hounsfield for the development of computed topographic scanning as the clinical realization of projection imaging (4).

Computed tomography is superior to sonography in diagnosis and management of abdominal masses. The exact origin of mass, size, shape and localization can be done with CT. Contrast enhanced CT scan helps in better localization, determining exact size of mass and degree of vascularity of the mass. Abdominal lymphadenopathy can also be better assessed (5). Presence of bowel gas and obesity does not have any hindrance in detection of the abdominal lesions in comparison to sonography. The disadvantages of computed tomography are high cost, ionization radiation and motion 
artifacts (6).

Magnetic Resonance Imaging (MRI) has become the primary diagnostic investigation for many clinical problems. Magnetic resonance imaging (MRI) of the abdomen is a proven and useful tool for the evaluation, assessment of severity, and follow-up of diseases of the abdomen.It provides excellentcontrast that can reveal subtle variations in tissues. MRI also provides a significant diagnostic rolein discrimination of inflammation from malignancy ortissue necrosis(7). No ionizing radiation or nephrotoxic contrast media isused, and the most commonly administered contrast agents, extracellular gadolinium (8).Gadolinium has a great number of MRI applications throughout the body.It is themost commonly used contrast agent for MRI, and several formulationsare currently available. As an intravenous contrast agent, it acts ina manner similar to that of the iodinated contrast agents used for CT. The development of fast MRI sequences has reduced acquisition times to the point that the entire liver can be imaged in 15 to 30 seconds (the length of time most people can hold their breath). Faster scanning is important because it makes possible acquisition of more images during a single period, reducingrespiratory and other motion artifacts Magnetic resonance images can be acquired in any orientation and, unlike CT scans, are not limited to the axial plane. The aim of this study was to assess and compare the precision of CT and MRI in detecting and differentiating variable causes of acute abdominal pain.

\section{MATERIALS AND METHODS}

In the current study, 15 patients ( 9 males, 6 females), aged 31 to 66 years who referred to the emergency department at Tripoli medical center during June 2016. Following various laboratory investigations, those patients who were suffering an acute abdomen had an erect and supine abdominal X-ray. All of them were recommended to have an abdominal CT examination, and then they also recommended havingan abdominal MRI for making the final differential diagnosis, because the abdominal X-ray result does not give them enough details about those cases. Even sometimes the result of abdominal X-ray was looks like normal. All those patients who already had abdominal X-ray result were prepared for abdominal and even pelvic CT studies with or without contrast according to the medical requests.

Before administration of CT and MRI for each patient, the research and its goals for each patient were explained. All patients signed the consent form for cooperating in the project. Those patients were examined with CT and MRI before surgery. Each patient underwent a preoperative MRI using a 3.0 Tesla superconductive MRI scanner (General Electric Vectra, IGF Medical, Milwaukee, Wisconsin, USA) focusing on the three-dimensional time-of-flight (3D-TOF) sequence. CT Scan Imaging was performed by GE HiSPEED NX/I Pro CT device. The images were taken in one radiological center and by one operator. In this way, we could omit the bias that originates from different machines and operators. Pathologists evaluated the lesions as benign or malignant, without any information about CT scan or MRI reports. CT scan reported cystic or solid lesion, with or without calcification and edema, shifting and enhancement. The MRI of the patients had beenperformed to evaluate the severity of acute abdominal pain or differential diagnosis, screening for abdominal lesions suspected with other imaging modalities. CT scan or MRI reports were concluded lesions as benign or malignant. All data were analyzed by SPSS software version 15 with fisher-exact test. Differences were considered significant in $\mathrm{P}$ value less than 0.05 .

\section{RESUlTS}

The clinical findings-based diagnosis rendered in patients with acute abdominal pain is often inaccurate. Therefore, imaging plays an important role in the treatment of patients with acute abdominal pain. To examine the effectiveness of different radiological diagnostic techniques in evaluating different causes of acute abdomen, a group of fifteen patients admitted at Tripoli medical center with acute abdomen. After performing X-rays for all patients, further radiological examinations using CT and MRI were applied for the quality of imaging and better differential diagnosis. The results showed that:in eight cases $(53.33 \%)$ of acute abdomen, magnetic resonance imaging (MRI) gave more details than computed tomography scans (CT); in three cases (20\%) of acute abdomen, computed tomography scans (CT) gave more details than magnetic resonance imaging (MRI); in four cases (26.66) of acute abdomen, the magnetic resonance imaging (MRI) and computed tomography scans (CT), gave the same information or details; in one case $(6.66 \%)$, the abdominal $\mathrm{X}$-ray result, and the computed tomography scan (CT) results were similar. All types of tumors within the abdominal cavity except hepatocellular carcinoma, MRI showed more imaging details than CT as shown in table (1)

Table 1

\begin{tabular}{|c|c|c|c|c|c|}
\hline \multirow[t]{2}{*}{ No } & \multirow[t]{2}{*}{ Age } & \multirow[t]{2}{*}{ Sex } & \multicolumn{2}{|c|}{ Result after seeing the Images } & \multirow{2}{*}{$\begin{array}{l}\text { Comparison of diagnosis } \\
\text { rating CT and MRI }\end{array}$} \\
\hline & & & Abdomen CT & Abdomen MRI & \\
\hline 1 & 32 & female & Insulinoma & Insulinoma & $\mathrm{X}$-ray $<\mathrm{CT}<\mathrm{MRI}$ \\
\hline 2 & 64 & male & Amebic liver abscess & Amebic liver abscess & $\mathrm{X}$-ray $<\mathrm{CT}=\mathrm{MRI}$ \\
\hline 3 & 54 & female & Renal vein thrombosis & Renal vein thrombosis & X-ray $<\mathrm{CT}<\mathrm{MRI}$ \\
\hline 4 & 57 & male & Hepatocellular carcinoma & Hepatocellular carcinoma & $\mathrm{X}$-ray $<\mathrm{CT}>\mathrm{MRI}$ \\
\hline
\end{tabular}


International Journal of New Technology and Research (IJNTR) ISSN: 2454-4116, Volume-5, Issue-8, August 2019 Pages 43-47

\begin{tabular}{|c|c|c|c|c|c|}
\hline 5 & 66 & male & Cholangiocarcinoma & Cholangiocarcinoma & $\mathrm{X}$-ray $<\mathrm{CT}<\mathrm{MRI}$ \\
\hline 6 & 42 & female & Bartholin's abscess & Bartholin's abscess & $\mathrm{X}$-ray $<\mathrm{CT}=\mathrm{MRI}$ \\
\hline 7 & 48 & male & Calcification & Calcification & $\mathrm{X}-\mathrm{ray}=\mathrm{CT}>\mathrm{MRI}$ \\
\hline 8 & 39 & male & Adrenal masses & Adrenal masses & $\mathrm{X}$-ray $<\mathrm{CT}<\mathrm{MRI}$ \\
\hline 9 & 52 & female & Ovarian cancer & Ovarian cancer & $\mathrm{X}$-ray $<\mathrm{CT}<\mathrm{MRI}$ \\
\hline & 58 & female & Pancreatic carcinoma & Pancreatic carcinoma & $\mathrm{X}$-ray $<\mathrm{CT}<\mathrm{MRI}$ \\
\hline $1^{1}$ & 44 & male & $\begin{array}{l}\text { Portal vein obstruction } \\
\text { (liver) }\end{array}$ & Bartholin's abscess & $\mathrm{X}$-ray $<\mathrm{CT}=\mathrm{MRI}$ \\
\hline $2^{1}$ & 64 & male & $\begin{array}{l}\text { Abdominal aortic } \\
\text { aneurysm }\end{array}$ & Abdominal aortic aneurysm & $\mathrm{X}$-ray $<\mathrm{CT}>\mathrm{MRI}$ \\
\hline & 47 & female & Pancreatic carcinoma & Pancreatic carcinoma & $\mathrm{X}$-ray $<\mathrm{CT}<\mathrm{MRI}$ \\
\hline $4^{1}$ & 53 & male & Retroperitoneal fibrosis & Retroperitoneal fibrosis & $\mathrm{X}$-ray $<\mathrm{CT}=\mathrm{MRI}$ \\
\hline $5^{1}$ & 31 & male & Pheochromocytoma & Pheochromocytoma & $\mathrm{X}$-ray $<\mathrm{CT}<\mathrm{MRI}$ \\
\hline
\end{tabular}

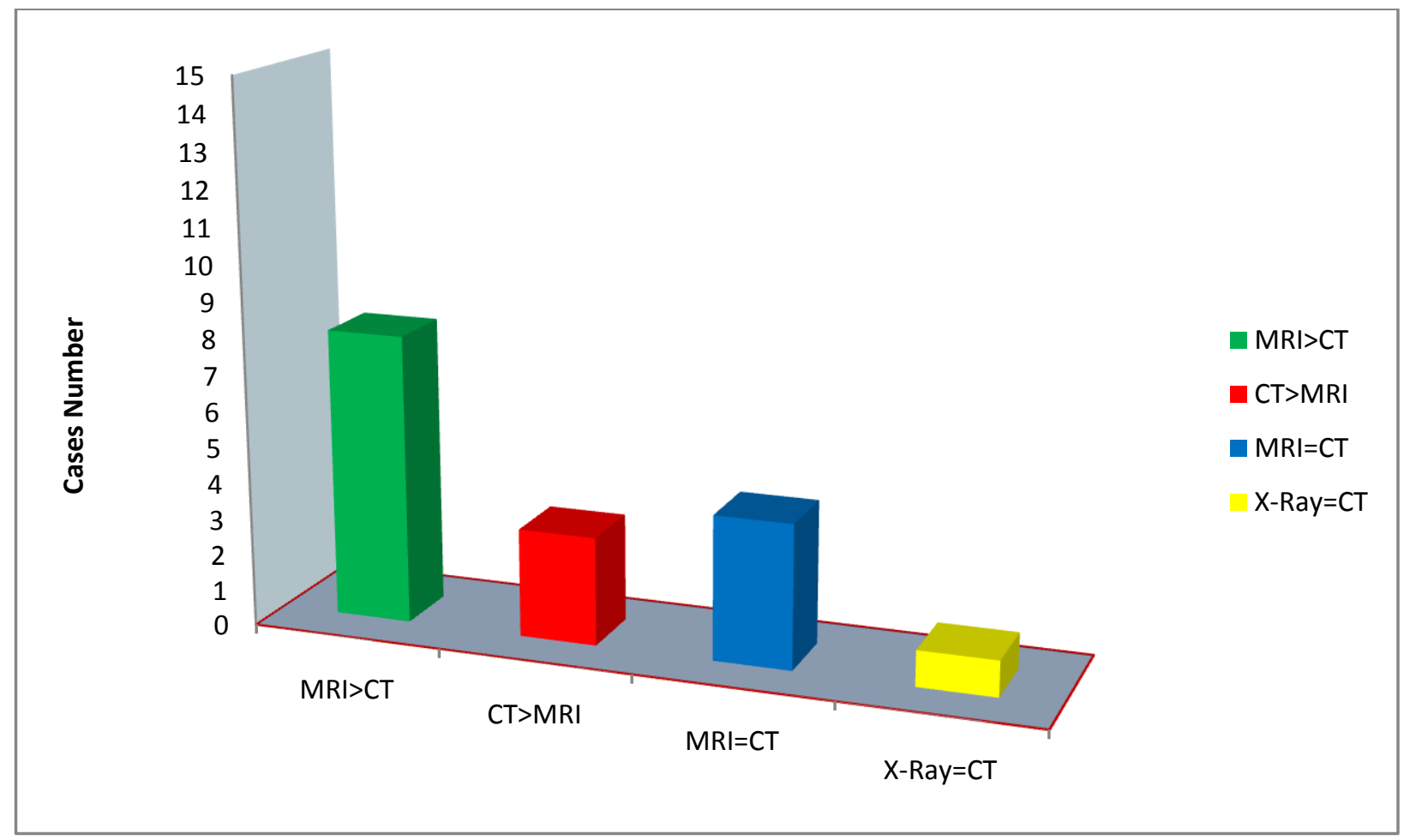

Figure 1.Comparison between CT and MRI in their ability to differentiate tumor-induced and non-tumor-induced acute abdominal pain. Figure shows that more $50 \%$ of cases, MRI is more sensitive to evaluate causes of abdominal pain than CT imaging technique. 


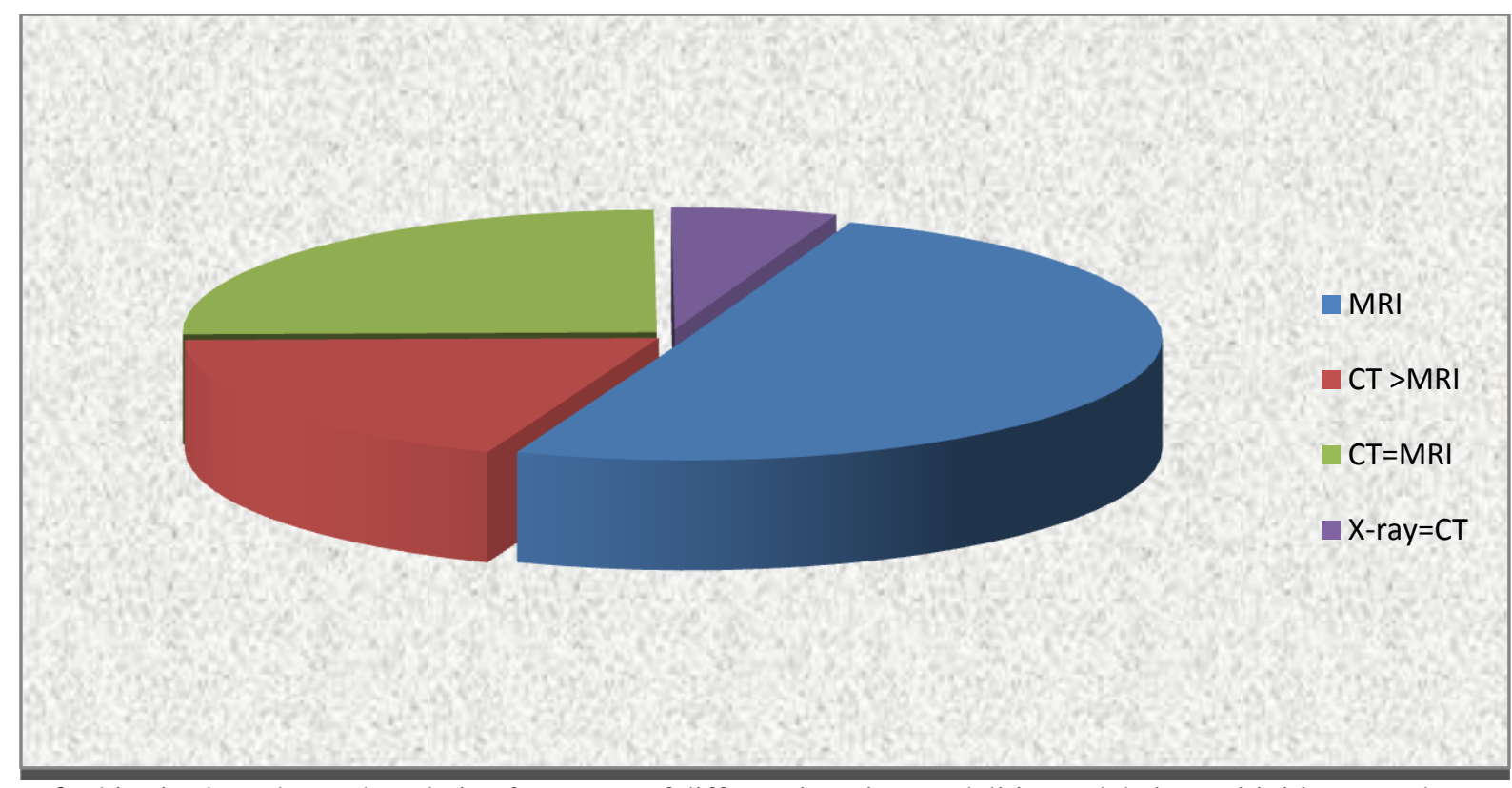

Figure 2.This pie chart shows the relative frequency of different imaging modalities and their sensitivities to evaluate causes of acute abdominal pain. MRI is more effective imaging tool in detecting and differentiating the cause of acute abdominal pain than any other imaging profile.

tumors, necrotic lesions and fibrosis, magnetic resonance imaging (MRI) gave more details than computed tomography

\section{DISCUSSION}

Calcification is a notable radiologic feature that occasionally observed in tumors, regardless of the organ or type of tumor. $(9,10)$. In clinical practice, CT is regarded as the gold standard for the identification of calcified lesions $(11,12)$.Some types of abdomen diseases cause calcium deposits (calcification) in the abdomen. Calcification appears in mucinous neoplasm of appendix, pancreatic neuroendocrine tumors up to $20 \%$ compared with to only $2 \%$ in pancreatic adenocarcinoma (13), and intratumoral calcification in case of biliary cystadenocarcinoma. In agreement with previous studies, our results showed that inthree cases, the computed tomography scan (CT) gave more details than MRI scan, and CT imaging is the first modality of choice to evaluate the calcification.

An axial contrast-enhanced CT image has demonstrated similar findings with MRI in case of aortic aneurysm with a wall calcification (14). Three cases of the present study (20\%) of acute abdomen, computed tomography scans (CT) gave more details than magnetic resonance imaging (MRI). The present study showed that CT was more effective in detecting aneurysm than MRI.It has been shown that a high diagnostic performance can be achieved using $\mathrm{CT}$ as a diagnostic tool in detecting bowel ischemia and venous thrombosis (15). The present study showed that MRI is an alternative imaging technique for evaluating acute abdomen caused by circulatory disturbances.It has been shown that magnetic resonance imaging (MRI) is the preferred way for evaluating soft tissue lesions, providing more information on hemorrhage, necrosis, edema, cystic and myxoid degeneration, and fibrosis(16). Moreover, MRI provides superior soft tissue resolutionthan any profile imaging, which can aid the assessment of extension and adjacent infiltration and lymph node involvement. In agreement with the previous study, the present study showed that more half of cases $(53.33 \%)$ of acute abdomen including abdominal and pelvic scans (CT). Previous studies have showed that MRI imaging is more accurate than CT scan for diagnosis brain tumors and biopsy correlation (17). In other studies, conducted by Fischer et al, have shown that contrast enhanced MRI is comparable or superior toot her imaging modalities such as CT and/or endoscopic retro grade cholangiography, pancreatography in patients with pancreatic tumors (18). In comparative studies performed by Schultz et alin the preoperative imaging of hepatic neoplasms to evaluate the diagnostic value of MRI and CT. The study concluded thatferumoxides-enhanced MRI is an economically feasible imaging methodthat will alter the clinical management in a substantial number ofpatients as compared with contrast-enhanced CT (19). Moreover, detection of hepatocellular carcinoma by MRI imaging has been shown to be improved using the subtraction of dynamic contrast enhanced series of MRI machine (20).Ultrafast MRI, and MRI enhanced with liver-specific contrast agents have allowed for better detection andcharacterization of focal hepatic neoplasms (21). However, the present study showed that CT scan had more imaging detail in detecting HCC than MRI. Disagreement could be attributed to either using different imaging protocol or different contrast medium or different tumor stage or mass detail at time of imaging. Siddiki et al have shown that MRI had more accurate examination for detecting abdominal and pelvic abscesses than any profile imaging (22). However, the present study has shown no significant difference in imaging detection of abdominal abscess either using MRI or CT scan.

\section{CONCLUSION}

Computed tomography (CT) can be used to detect calcifications and bone erosion which could not be seen on radiographs. Magnetic resonance imaging (MRI) is the preferred way for evaluating soft tissue lesions and provides information on hemorrhage, necrosis, and fibrosis. MR 
imaging may be used as a second-line imaging strategy if CT is not able to solve the diagnostic problem, particularly in tumor-caused abdominal pain with lymph node involvement.

\section{ACKNOWLEDGMENTS}

All authors contributed to the manuscript.

\section{REFERENCES}

[1] Powers JH. Acute appendicitis during the later decades of life: some remarks on the incidence of the disease in a rural area. Ann Surg 1943; 117:221-233.

[2] Koh DM, Collins DJ. Diffusion-weighted MRI in the body: applications and challenges in oncology. AJR Am J Roentgenol 2007;188(6):1622-1635.

[3] MacKersie AB, Lane MJ, Gerhardt RT et al. Nontraumatic acute abdominal pain: unenhanced helical CT compared with three-view acute abdominal series. Radiology2005; 237:114-122.

[4] Hendee WR. Cross Sectional Medical Imaging: A History. Radiographics 1989; 9: 1155-80

[5] Tevfik A, Mustafa K, Ufuk U, İrfan HA, Osman İ. Retroperitoneal ganglioneuroma. FakDerg. 2009; 26(2): 163-65.

[6] 6. Pablo RR, Robert LB, Dennis F, Spencer BG, Seth NG, Jay PH. ACR appropriate criteria-Acute Pancreatitis-2006: 1-5.

[7] GROSSMARN. I., JOSEPHP. M. and WOLFG.: Experimental intracranial septic infarction. Magnetic resonance enhancement. Radiology 155 (1985), 649.

[8] Prince MR, Arnoldus C, Frisoli JK: Nephrotoxicity of high-dose gadolinium compared with iodinated contrast. J MagnReson Imaging 6:162-166, 1996

[9] Curry CA, Eng J, Horton KM, Urban B, Siegelman S, Kuszyk BS, et al. CT of primary cystic pancreatic neoplasms: can CT be used for patient triage and treatment? AJR Am J Roentgenol. 2000; 175:99-103.

[10] Ko EY, Ha HK, Kim AY, Yoon KH, Yoo CS, Kim HC, et al. CT differentiation of mucinous and nonmucinous colorectal carcinoma. AJR Am J Roentgenol. 2007; 188:785-791.

[11] Izawa N, Sawada T, Abiko R, Kumon D, Hirakawa M, Kobayashi M, et al. Gastrointestinal stromal tumor presenting with prominent calcification. World J Gastroenterol. 2012; 18:5645-5648.

[12] Baek JH, Lee JM, Kim SH, Kim SJ, Kim SH, Lee JY, et al. Small (<or=3 cm) solid pseudopapillary tumors of the pancreas at multiphasic multidetector CT. Radiology. 2010; 257:97-106.

[13] Giachelli CM. Ectopic calcification: gathering hard facts about soft tissue mineralization. Am J Pathol. 1999; 154:671-675.

[14] Agarwal A, Yeh BM, Breiman RS, Qayyum A, Coakley FV. Peritoneal calcification: causes and distinguishing features on CT. AJR Am J Roentgenol. 2004; 182:441-445.

[15] Akira Furukawa ${ }^{1}$, Shuzo Kanasaki, NaoakiKono, Makoto Wakamiya. CT Diagnosis of Acute Mesenteric Ischemia from Various Causes. American Journal of Roentgenology. 2009;192: 408-416.

[16] Jingfeng Zhang, ${ }^{1}$ Yanyuan $\mathrm{Li},{ }^{2}$ Yilei Zhao, ${ }^{1}$ and JianjunQiao. 2018. CT and MRI of superficial solid tumors. Quant Imaging Med Surg. 2018; 8(2): 232-251

[17] TaghipourZahir SH MD1, Rezaei sadrabadi M MD2, Dehghani F MD3. 2011. Evaluation of Diagnostic Value of CT Scan and MRI in Brain Tumors and Comparison with Biopsy. Iranian Journal of Pediatric Hematology Oncology Vol 1. No 4.

[18] Fischer U,VosshenrichR, Horstmann O, Becker H, Salamat B, BaumF, Grabbe E (2002). Preoperative local MRI staging of patients with suspected pancreatic mass. Eur Radio. 112:296-303

[19] Schultz JF, Bell JD, Goldstein RM, et al: Hepatic tumor imaging using iron oxide MRI: Comparison with computed tomography, clinical impact, and cost analysis. Ann Surg Oncol 6:691-698, 1999

[20] 20- Mustafa Seçil, FundaObuz, Canan Altay, mürGencel, Enisİ̆gci, zgülSağol, OğuzDicle. 2008The role of dynamic subtraction MRI in detection of hepatocellular carcinoma. Diagn Inter. Radio. 1 2008; 14:200-204

[21] James P. Earls, MD. Comparison Studies of CT and MRI in Patients with Hepatic Metastases. ONCOLOGY 2000; 14(Suppl 3): 21-28,

[22] Siddiki H, Fidler J. MR imaging of the small bowel in Crohn's disease. $\begin{array}{llll}\text { Eur } & \text { J } & \text { Radio. } & \text { 12009; }\end{array}$ 